\title{
Electrical interaction between two cylinders with an ion-penetrable charged membrane in an oil/water interface
}

\author{
Jyh-Ping Hsu ${ }^{\mathrm{a}, *}$, Jian-Hong Lu ${ }^{a}$, Yung-Chih Kuo ${ }^{\mathrm{b}}$, Shiojenn Tseng ${ }^{\mathrm{c}}$ \\ a Department of Chemical Engineering, National Taiwan University, Taipei, Taiwan 10617, Republic of China \\ ${ }^{\mathrm{b}}$ Department of Chemical Engineering, Eastern College of Technology and Commerce, 110 Tung-Fung Rd., Hu-Nei, Kaohsiung, \\ Taiwan 829, Republic of China \\ ${ }^{\mathrm{c}}$ Department of Mathematics, Tamkang University, Tamsui, Taipei 25137, Republic of China
}

Received 24 July 2000; accepted 17 August 2000

\begin{abstract}
The electrical interaction between two long, parallel cylinders each is covered by an ion-penetrable charged membrane immersed in an oil/water interface is investigated. The effects of contact angle, radius of cylinder, and membrane thickness on the electrical interaction force are examined. The results of numerical simulation reveal that the following conditions lead to a greater electrical interaction force: (i) a larger contact angle, i.e. a larger fraction of a cylinder in the oil phase; (ii) a larger cylinder radius; and (iii) a thinner membrane. For a fixed ionic strength, the electrical interaction force is insensible to the type of electrolytes in the water phase, in general. However, if two cylinders are close enough, then the higher the valence of counterions the greater the electrical interaction force. (C) 2001 Elsevier Science B.V. All rights reserved.
\end{abstract}

Keywords: Electrical interaction; Two parallel cylinders; Ion-penetrable membrane; Oil/water interface

\section{Introduction}

Colloidal particles are ubiquitous in both human life and industrial processes. Typical example varies from biological entities such as blood cells and microorganisms to inorganic entities such as silicon oxide particles used in semi-conductor processing. Apparently, the behavior of a colloidal dispersion is closely related to both colloid-colloid and colloid-solvent interactions. Previous ef-

\footnotetext{
* Corresponding author. Fax: + 886-2-23623040.

E-mail address: jphsu@ccms.ntu.edu.tw (J.-P. Hsu).
}

forts on the relevant studies are mainly focused on the case of a single dispersed medium. The potential applications of a colloidal dispersion in various fields, however, have triggered the investigations on problems and phenomena, which involve two dispersion media, in the past two decades.

Emulsion is one of the most significant colloidal dispersions in practice. Water-in-oil emulsion, for example, can be found in oil recovery process. Here, since the formation of water drops may cause various problems [1], the goal is to create an unstable dispersion. Another example is water-in- 
oil emulsion, which can be used to produce nanometer sized metallic and semiconductor particles. In this case since the water drops play the role of micro chemical reactors, the task now is to create environment such that a stable dispersion is obtained. This can be achieved by dispersing fine entities such as surfactants, over the liquid-liquid interface [2]. Tadros and Vincent [3] concluded that emulsions formed by smaller particles and particles with a rougher surface, which are capable of forming an appropriate contact angle with liquid-liquid interface, are more stable. These findings were explained by Denkov et al. [4] through considering a mechanism, which takes the effect of contact angle hysteresis on the effective disjoining pressure isotherms into account. In medical and pharmaceutical applications, emulsions have been used to stabilize some hydrophobic drugs. Since emulsions are thermodynamically unstable, the goal here is to maintain their stability. Shi et al. [5] showed that bovine submaxillary gland mucin (BSM) is capable of increasing the stability of an emulsion.

Lyne et al. [6] examined the electrostatic interaction force between two identical, infinitely long, parallel cylinders in an oil-water interface for the case of symmetric electrolytes at three oil/water ratios. The Poisson-Boltzmann equation governing the spatial variation of electrical potential was solved numerically. The results obtained reveal that the repulsive electrical double layer force in the water phase is of short range nature and that in the oil phase is of long range nature. Hsu and Liu [7] discussed the electrostatic interaction between two long, parallel rod-like objects in an oil-water interface. Under the Debye-Huckle condition the governing Poisson-Boltzmann equation was solved analytically by a Green function method. They found that the thicker the double layer the more stable the system under consideration. For a fixed double layer thickness the closer the objects to cylinders or the larger the fraction of the objects in the water phase, the more stable the system.

In the present study, the electrostatic interaction between two long, parallel cylinders each is covered by an ion-penetrable charged membrane immersed in an oil/water interface is investigated. In particular, the effects of contact angle, radius of cylinder, and membrane thickness on the electrical interaction force are examined. Typical application of the problem under consideration is in oil-in-water and water-in-oil emulsions where a dispersed system is stabilized by adsorbing fine particles on the liquid-liquid interface. The adsorbed particles are usually charged, and form a thin layer on the interface. Biological system is another example in which biocolloids can be simulated by a rigid core covered by a charged membrane layer. The interaction between micelles and an interface belongs to this category. The problem considered in this study can also be used to

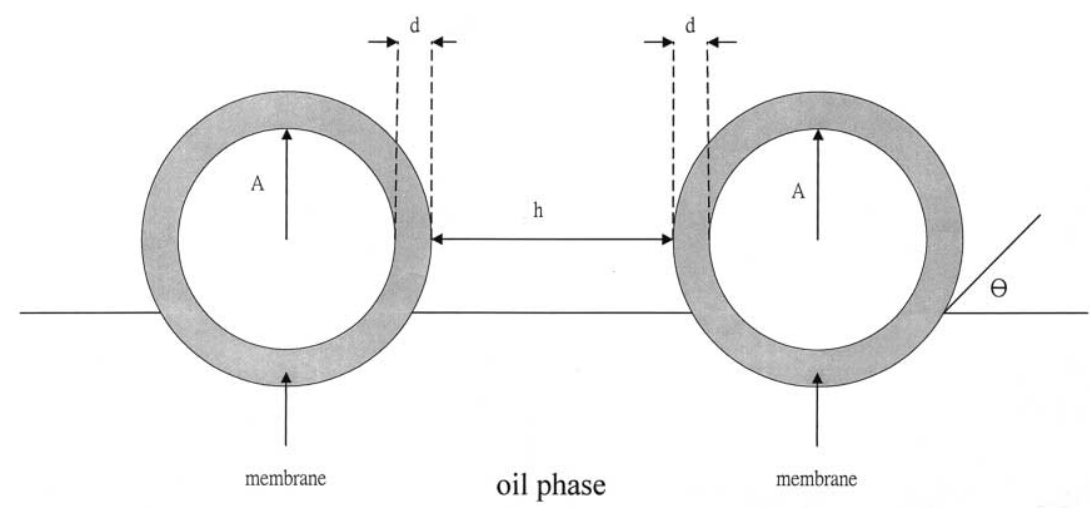

Fig. 1. Schematic representation of the system under consideration. Two identical, parallel long cylinders of radius $A$ are immersed in an oil-water interface with contact angle $\theta$. Each cylinder is covered by an ion-penetrable membrane of thickness $d$. $h$ is the closest surface-to-surface distance between two cylinders. 

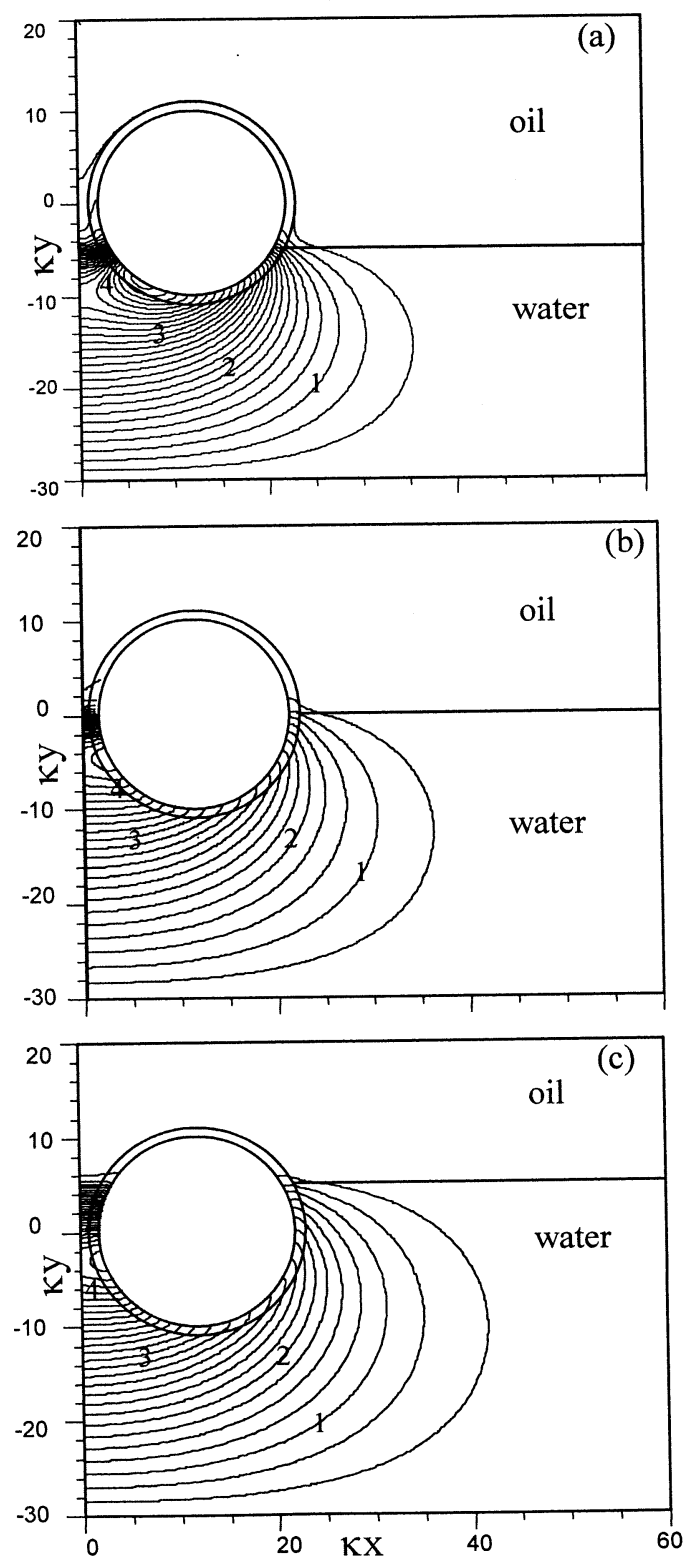

Fig. 2. Typical contours for the scaled electrical potential for the case $\kappa h=1.0$. (a) $\theta=60^{\circ}$, values for the scaled potential are (1) -0.3 ; (2) -0.9 ; (3) -1.6 ; (4) -2.6 ; (b) $\theta=90^{\circ}$, values for the scaled potential are (1) -0.4 ; (2) -1.2 ; (3) -2.6 ; (4) -3.0 ; (c): $\theta=120^{\circ}$ values for the scaled potential are (1) -0.6 ; (2): -1.4 ; (3) -2.8 ; (4) -4.2 . Key: $a: b=1: 1$, $I=2 \times 10^{-3} \mathrm{M}, C_{a}^{0}=2 \times 10^{-3} \mathrm{M}, \kappa A=10, \kappa d=1, z=1$, $\varepsilon_{r}=78$, and $T=298 \mathrm{~K}$.

simulate the interactions between surfactants, emulsifiers, and bio-molecules on a liquid-liquid interface. For instance, it is known that the efficiency of a tertiary oil recovery process can be raised by the addition of an appropriate amount of emulsifiers. Here, the interaction between
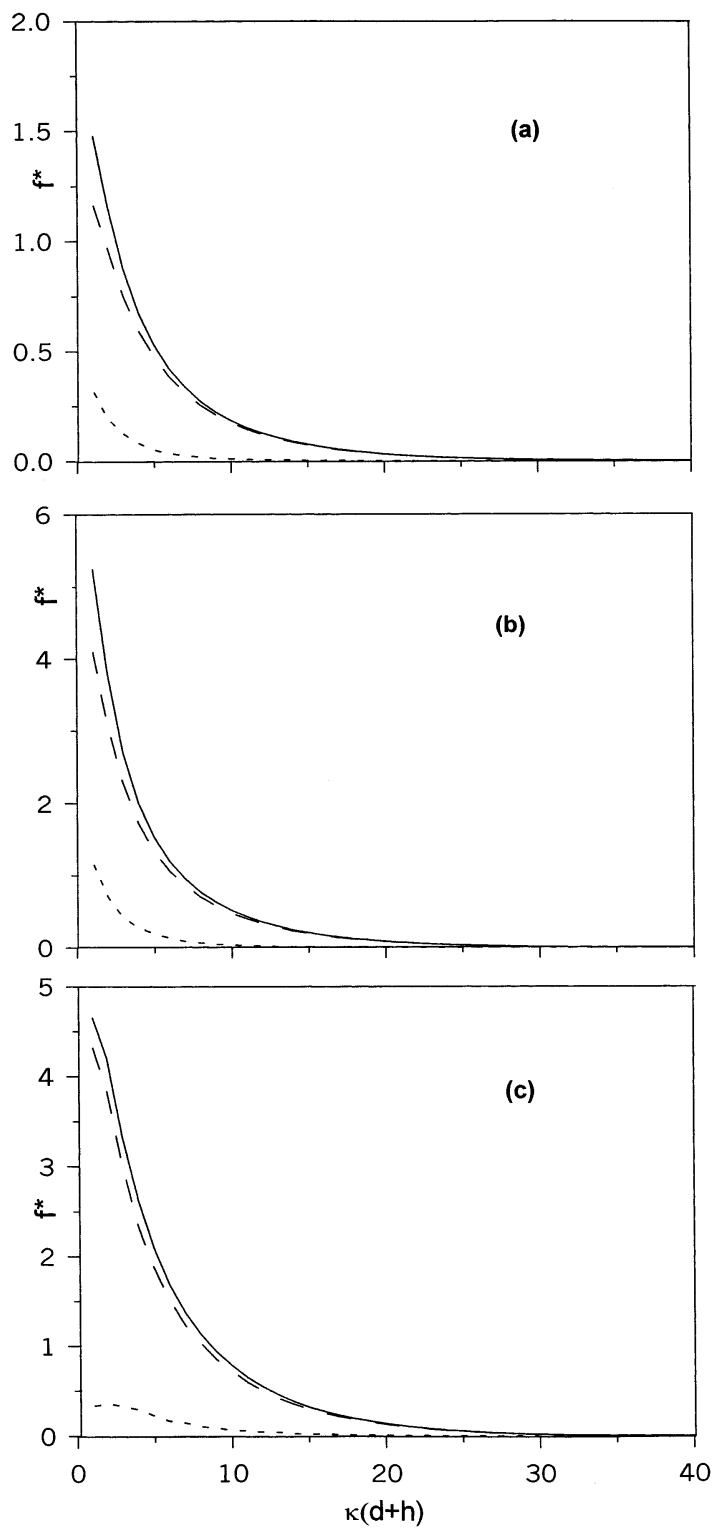

Fig. 3. Variation of scaled electrical interaction force between two cylinders $f^{*}$ as a function of the scaled separation distance between two cylinders $\kappa(d+h) . \theta=60^{\circ}$, (a), $\theta=90^{\circ}$, (b), and $\theta=120^{\circ}$, (c). Oil phase, - -, water phase, - - total, - - . Key: same as Fig. 2. 


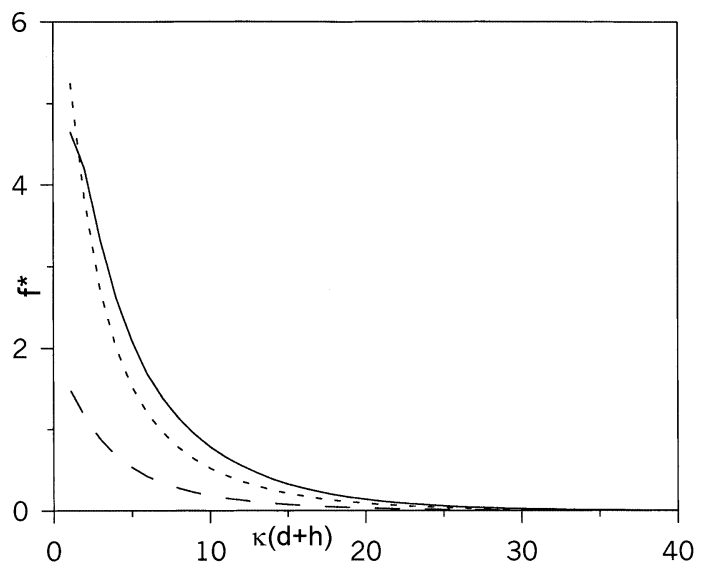

Fig. 4. Variation of scaled total electrical interaction force between two cylinders $f^{*}$ as a function of the scaled separation distance between two cylinders $\kappa(d+h)$ at various contact angles $\theta$ for the case of Fig. 3. $\theta=60^{\circ},--, \theta=90^{\circ},--$, and $\theta=120^{\circ}$, - -

emulsifiers on oil-liquid interface plays a significant role.

\section{Analysis}

Referring to Fig. 1, we consider two identical, parallel long cylinders of radius $A$ each is covered by an ion-penetrable membrane of thickness $d$ in an oil-water interface. Suppose that the membrane layer carries uniformly distributed negative fixed charge of valence $z$ and concentration $C_{0}$. Let $h$ be the closest surface-to-surface distance between two cylinders. The fraction of a cylinder in the oil phase is determined by the contact angle $\theta$. If $\theta>90^{\circ}$, then the faction of a cylinder in the oil phase is larger than that in the water phase, and the reverse is true if $\theta<90^{\circ}$. If $\theta=90^{\circ}$, the faction of a cylinder in the oil phase is the same as that in the water phase. For simplicity, we assume that the oil-water interface is flat. Suppose that the water phase contains $a: b$ electrolyte, and the oil phase is electrolyte free. In the water phase, if the spatial concentration of mobile ions follows the Boltzmann distribution, then the spatial electrical potential at equilibrium, $\varphi$, can be described by

$$
\begin{aligned}
\nabla^{2} \varphi & =\frac{a C_{a}^{0} F}{\varepsilon_{0} \varepsilon_{r}}\left[\exp \left(\frac{b e \varphi}{k_{\mathrm{B}} T}\right)-\exp \left(\frac{-a e \varphi}{k_{\mathrm{B}} T}\right)\right]+\frac{z F C_{0}}{\varepsilon_{0} \varepsilon_{r}} \\
A & <r<D \\
\nabla^{2} \varphi & =\frac{a C_{a}^{0} F}{\varepsilon_{0} \varepsilon_{r}}\left[\exp \left(\frac{b e \varphi}{k_{\mathrm{B}} T}\right)-\exp \left(\frac{-a e \varphi}{k_{\mathrm{B}} T}\right)\right] \\
D & \leq r<\infty
\end{aligned}
$$
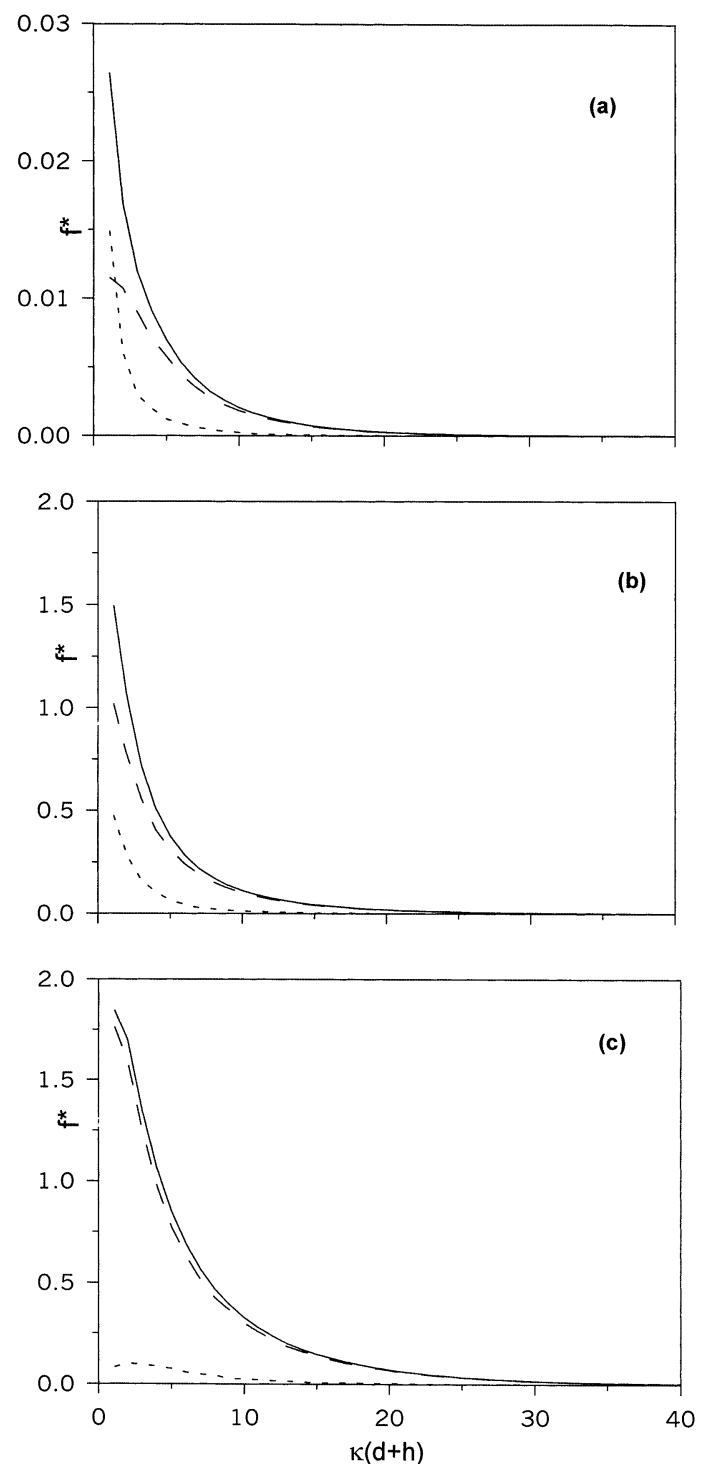

Fig. 5. Variation of scaled electrical interaction force between two cylinders $f^{*}$ as a function of the scaled separation distance between two cylinders $\kappa(d+h) . \theta=60^{\circ}$, (a), $\theta=90^{\circ}$, (b), and $\theta=120^{\circ}$, (c). Oil phase, - -, water phase, - - , total, - - . Key: same as Fig. 2 except that $\kappa A=5$. 


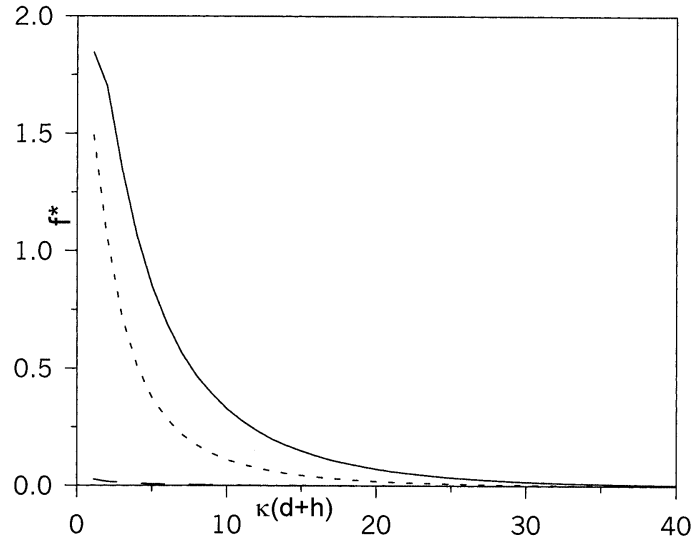

Fig. 6. Variation of scaled total electrical interaction force between two cylinders $f^{*}$ as a function of the scaled separation distance between two cylinders at various contact angles for the case of Fig. 5. $\theta=60^{\circ},--, \theta=90^{\circ},--$, and $\theta=120^{\circ}$, -

where $C_{a}^{0}$ is the bulk concentration of cations, $k_{\mathrm{B}}$ is the Boltzmann constant, $r$ is the radial distance, $\varepsilon_{0}$ and $\varepsilon_{r}$ are respectively the permittivity of a vacuum and the relative permittivity of the liquid phase, $T$ is the absolute temperature, $F$ and e are respectively the Faraday constant and the elementary charge, $\nabla^{2}$ is the Laplace operator, and $D=$ $A+\mathrm{d}$. Since electrolyte is absent in the oil phase, the distribution of electrical potential is described by

$\tilde{\nabla}^{2} \varphi=\frac{z F C_{0}}{\varepsilon_{0} \varepsilon_{r}}, \quad A<r<D$

$\tilde{\nabla}^{2} \varphi=0, \quad D \leq r<\infty$

In terms of scaled variables, Eqs. (1) through (4) can be expressed as

$\tilde{\nabla}^{2} \psi=\frac{g+i N}{a+b}, \quad \kappa A<\kappa r<\infty$, water phase

$\tilde{\nabla}^{2} \psi=\frac{i N}{a+b}, \quad \kappa A<\kappa r<\infty$, oil phase

where $\psi=e \varphi / k_{\mathrm{B}} T, \quad \kappa^{2}=e^{2} a(a+b) C_{a}^{0} / \varepsilon_{0} \varepsilon_{r} k_{\mathrm{B}} T$, $g=\exp (b \psi)-\exp (-a \psi), N=z C_{0} / a C_{a}^{0}=(a+b)$ ${ }_{z} C_{0} / 2 I, I$ being the ionic strength, $\tilde{\nabla}^{2}$ is the scaled Laplace operator, and $i$ is a region index $(i=0$ denotes liquid phase, $i=1$ represents membrane phase). The following boundary conditions are assumed:

$\psi \rightarrow 0$ as $\kappa r \rightarrow \infty$
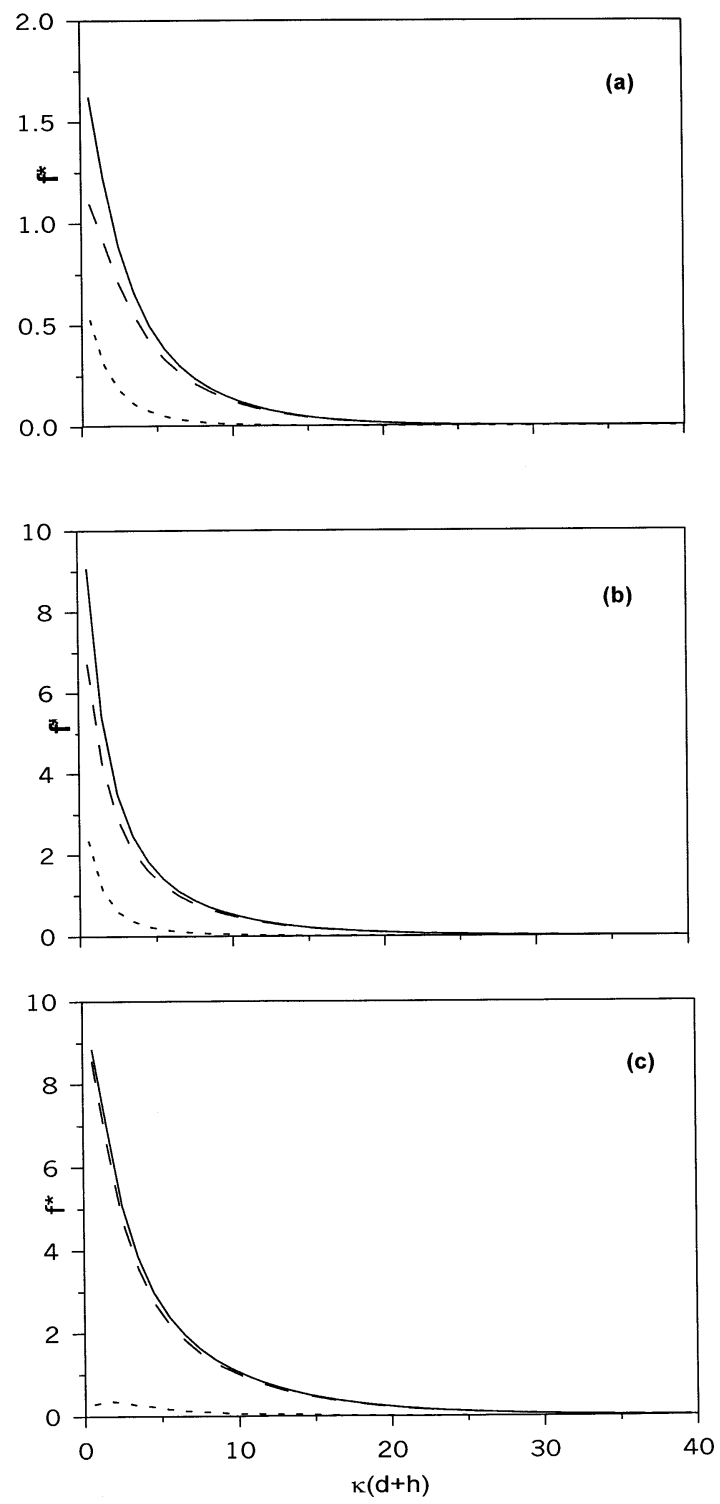

Fig. 7. Variation of scaled electrical interaction force between two cylinders $f^{*}$ as a function of the scaled separation distance between two cylinders $\kappa(d+h) . \theta=60^{\circ}$, (a), $\theta=90^{\circ}$, (b), and $\theta=120^{\circ}$, (c). Oil phase, - -, water phase, - - total, - - . Key: same as Fig. 2 except that $\kappa d=0.5$. 


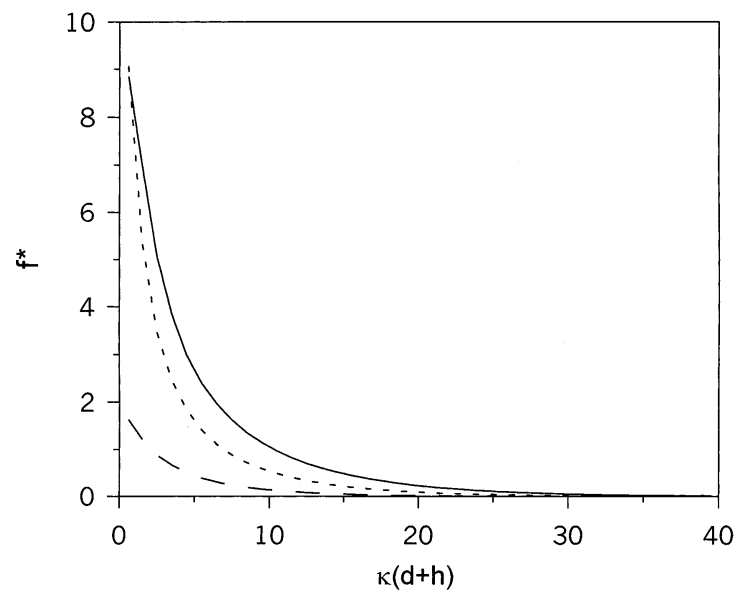

Fig. 8. Variation of scaled total electrical interaction force between two cylinders $f^{*}$ as a function of the scaled separation distance between two cylinders $\kappa(d+h)$ for the case of Fig. 7.

$\frac{\partial \psi}{\partial n}=0 \quad$ at $\kappa r=\kappa A$

$\varepsilon_{0} \frac{\partial \psi}{\partial n}=\varepsilon_{w} \frac{\partial \psi_{w}}{\partial n} \quad$ and

$\psi_{0}=\psi_{w}$ at oil water interface

$$
\begin{gathered}
\left.\left(\frac{\partial \psi}{\partial n}\right)\right|_{\kappa r \rightarrow \kappa D^{+}}=\left.\left(\frac{\partial \psi}{\partial n}\right)\right|_{\kappa r \rightarrow \kappa D^{-}} \text {and } \\
\left.\psi\right|_{\kappa r \rightarrow \kappa D^{+}}=\psi_{\kappa r \rightarrow \kappa D-}
\end{gathered}
$$

where $n$ is the unit normal vector and subscripts o and $\mathrm{w}$ denote oil and water phases respectively. The electrical interaction force between two cylinders can be evaluated by [6]

$$
\begin{aligned}
f= & \int_{-a \cos \theta}^{\infty}\left[\left(\frac{\varepsilon_{w}}{8 \pi}\right)\left(\frac{\partial \varphi}{\partial y}\right)^{2}+\Pi\right] \mathrm{d} y \\
& -\left(\frac{\varepsilon_{0}}{8 \pi}\right) \int_{-\infty}^{-a \cos \theta}\left(\frac{\partial \varphi}{\partial y}\right)^{2} \mathrm{~d} y,
\end{aligned}
$$

where $y$ is the axis perpendicular to the oil-water interface and on the mid-plane between two cylinders, and $\Pi$ is osmotic pressure. For $a: b$ electrolytes, we have

$$
\prod=k_{\mathrm{B}} T C_{a}^{0}\left[\frac{b \exp (-a \varphi)+a \exp (b \varphi)}{a}-\frac{2 b}{a}\right] .
$$

For convenience, we define the scaled electrical interaction force $f^{*}$ as

$f^{*}=\left(\frac{8 \pi f}{\varepsilon_{w}}\right)\left(\frac{e}{k_{\mathrm{B}} T}\right)^{2}$
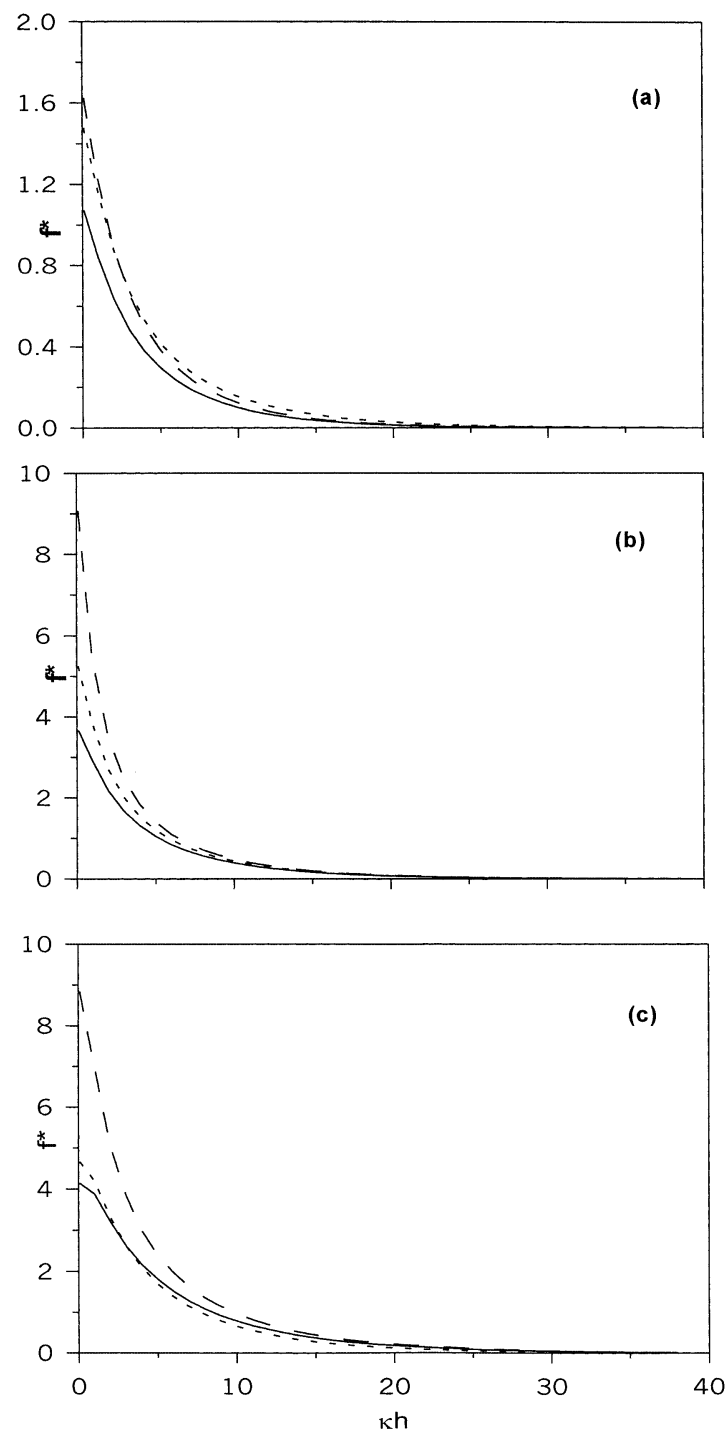

Fig. 9. Variation of scaled total electrical interaction force between two cylinders $f^{*}$ as a function of the scaled separation distance between two cylinders $\kappa h$ ) for various $\kappa d$. The total amount of fixed charge in the membrane remains constant. - -, $\kappa d=5,--, \kappa d=7.5,--, \kappa d=10 . \theta=60^{\circ},(\mathrm{a}), \theta=90^{\circ}$, (b), and $\theta=120^{\circ}$, (c). Key: same as Fig. 2 except that $\kappa \mathrm{d}$ is varying. 
The governing equations for the electrical potential are solved numerically by PDEase2D [8], which is based on the finite element method with Galerkin weighted residuals

\section{Results and discussion}

Fig. 2 shows the typical contours for the scaled electrical potential at three different contact angles. This figure reveals that the decrease in electrical potential as a function of distance in the oil phase is much slower than that in the water phase which implies that the electrical interaction between two particles in the water phase is shortranged, and that in the oil phase is long-ranged. This is consistent with the result of Lyne et al. [6]. The simulated variation in the scaled electrical interaction force between two cylinders $f^{*}$ as a function of the scaled separation distance between two cylinders $\kappa(d+h)$ at various contact angles is presented in Fig. 3, and the corresponding variation in the total electrical interaction force between two cylinders as a function of $\kappa(d+h)$ is summarized in Fig. 4. As can be seen from Fig. 3, the contribution to the scaled interaction force by the oil phase is greater than that by the water phase. It is interesting to note that for the case $\theta=120^{\circ}$, the electrical interaction force contributed by the water phase has a local maximum as $\kappa(d+h)$ varies. Fig. 4 suggests that if $\kappa(d+h)$ is large, the total electrical interaction force between two cylinders increases with contact angle, that is, the larger the volume fraction of a cylinder in the oil phase, the greater the electrical interaction force. This, however, may not be true if $\kappa(d+h)$ is small. This can be inferred from Fig. 3 in which the double layer interaction in the water phase is stronger at a smaller contact angle. Similar conclusions as those drawn from Fig. 3 and Fig. 4 for the case of 1:1 electrolytes can be obtained for the case of $2: 1$ and 3:1 electrolytes.

Fig. 5 illustrates the variation in the scaled electrical interaction force between two cylinders $f^{*}$ as a function of the scaled separation distance between two cylinders $\kappa(d+h)$ at various contact angles for the case of a smaller $\kappa A$ (i.e. smaller cylinders) than that of Fig. 3. The corresponding variation in the total electrical interaction force between two cylinders as a function of $\kappa(d+h)$ is summarized in Fig. 6. Note that, as can be seen from Fig. 5, for a small $\theta$ if $\kappa(d+h)$ is small, the electrical interaction force contributed by the water phase is larger than that by the oil phase. This is also observed by Lyne et al. [6], where rigid cylinders at constant surface potential is considered. Similar to Fig. 4, the total electrical interaction force between two cylinders increases with the volume fraction of a cylinder in the oil phase (or $\theta$ ), as can be seen in Fig. 6. However, the total electrical interaction force at a small $\kappa(d+h)$ for the case when $\theta=90^{\circ}$ does not exceeds that when $\theta=120^{\circ}$. A comparison between Fig. 4 and Fig. 6 reveals that the electrical interaction force between two cylinders increases with the radius of a cylinder. This is because the larger the radii of the cylinders the wider the interaction range of the electrostatic repulsion between them.

The variation in the scaled electrical interaction force between two cylinders $f^{*}$ as a function of the scaled separation distance between two cylinders $\kappa(d+h)$ at various contact angles for the case of a smaller $\kappa d$ (i.e. thinner membrane layer) than that of Fig. 3 is shown in Fig. 7. The corresponding variation in the total electrical interaction force between two cylinders as a function of $\kappa(d+h)$ is summarized in Fig. 8. Here, the total amount of fixed charge in the membrane layer is the same as that of Fig. 3. Similar conclusions as those drawn from Fig. 3 and Fig. 4 can be obtained from Fig. 7 and Fig. 8. Intuitively, it is expected that the thinner the membrane the greater the electrical interaction force between two cylinders since for a constant total amount of fixed charge, the thinner the membrane the higher the fixed charge density, and the stronger the induced electric field. However, as illustrated in Fig. 9, where the variations in the scaled total electrical interaction force between two cylinders as a function of $\kappa h$ at various contact angles for various $\kappa \mathrm{d}$ are presented, this is only correct for $\theta=90^{\circ}$. For both $\theta=60^{\circ}$ and $120^{\circ}$ it is correct only for small separation distance between two cylinders; for an arbitrary separation distance, no general rule can be obtained. 


\section{Acknowledgements}

This work is partially financially supported by the National Science Council of the Republic of China.

\section{References}

[1] W.I. Friesen, S. Levine, J. Colloid Interface Sci. 150 (1992) 517.
[2] S.U. Pickering, J. Chem. Soc. 91 (1907) 2001.

[3] Th.V. Tadors, B. Vincent, Encycl. Emulsion Technol. 1 (1983) 129.

[4] N.D. Denkov, I.B. Ivanov, P.A. Kralchevsky, D.T. Wasan, J. Colloid Interface Sci. 150 (1992) 589.

[5] L. Shi, C. Miller, K.D. Caldwell, P. Valint, Colloids Surf. B 15 (1999) 303.

[6] M.D. Lyne, B.D. Bowen, S. Levine, J. Colloid Interface Sci. 150 (1992) 374.

[7] J.P. Hsu, B.T. Liu, J. Phys. Chem. B 101 (1997) 5147.

[8] PDEase2D Reference Manual, 3rd Edn., November 1996. 